\title{
Atividade física e mental no risco de demência em idosos
}

Physical and mental activity on the risk of dementia in the elderly

Andy Petroianu', Henrique Xavier de Miranda Capanema', Mariana Moura Quintão Silva', Nathália Teixeira Palla Braga'

\section{RESUMO}

Objetivo: Este trabalho visa a verificar se de fato existe relação entre o risco de demência e a falta de estímulo físico ou mental em idosos. Métodos: Trezentos e três idosos, com idade de 80 anos ou mais, foram estudados por meio de questionários específicos e distribuídos em três grupos de acordo com a prática de atividade física ou mental. Todos foram submetidos ao Miniexame do Estado Mental (MEEM) e, a partir da pontuação obtida, considerando diferentes pontos de corte, de acordo com a escolaridade, foi comparado o risco de desenvolvimento de demência entre os grupos. Resultados: Não houve diferença da pontuação entre sexos. Comparando as incidências de risco aumentado de demência, os indivíduos que não praticavam atividade alguma tiveram risco relativo de 4,27, quando comparados com os indivíduos que praticavam atividade mental, e de 2,21, quando comparados aos praticantes de atividade física. Esses últimos tiveram risco relativo de 1,93, em relação aos praticantes de atividade mental. Conclusão: A prática regular de atividades físicas e mentais retarda 0 declínio cognitivo, reduzindo o risco de demência. Entre essas atividades, as mentais foram mais eficazes.

\section{ABSTRACT}

Objective: This study aims to verify if there is a relation between risk of dementia and the absence of physical or mental stimulation in the elderly. Methods: Three hundred and three elderly people with the age of 80 years old or over, were assessed by means of the specific questionnaires. They were divided into three groups according to practice of physical or mental activity. They were submitted to the Mini-Mental State Examination (MMSE) and assessed according to their educational level and the risk of dementia. Results: There was not difference in scores between the genders. When comparing the increased risk of dementia incidence, individuals who did not practice any activity had a 4.27 relative risk when compared to individuals who practiced mental activity, and 2.21 when compared to practitioners of physical activity. The practitioners of physical activity had a 1.93 relative risk in relation to practitioners of mental activity. Conclusion: The regular practice of physical and mental activities delays the cognitive decline, reducing the risk of dementia. The mental activity was more effective.

\section{Keywords}

Aged, 80 and over, dementia, motor activity, leisure activity.
1 Universidade Federal de Minas Gerais (UFMG), Departamento de Cirurgia da Faculdade de Medicina. 


\section{INTRODUÇÃO}

De acordo com dados do IBGE'1, no Brasil, em 2006, o número de pessoas com mais de 60 anos superou 19 milhões, o que corresponde a mais de 10\% da população. Esse é o grupo com o maior crescimento, destacando pessoas com mais de 80 anos, que totalizavam 2,4 milhões naquele ano. Esse aumento da idade média da população traz como consequência maior incidência de doenças crônico-degenerativas, sendo a demência responsável por grande impacto na vida dos pacientes e de seus familiares ${ }^{2}$.

Durante o envelhecimento, ocorrem diversas alterações no sistema nervoso central, como redução do peso encefálico, do fluxo sanguíneo cerebral e do número de neurônios e lentificação da velocidade da condução nervosa, entre outras. Em consequência, observam-se declínios graduais nas funções cognitivas, considerados comuns, como a perda da memória e até déficits mentais mais acentuados. O lobo frontal parece ser a região mais acometida, principalmente em sua porção dorsolateral, a qual é responsável pela realização de tarefas dependentes da função executiva e da memória de trabalho. Clinicamente, observam-se lentificação do processamento cognitivo, redução da atenção, dificuldade no resgate das informações aprendidas, diminuição da memória prospectiva e da memória contextual. Tais alterações não trazem prejuízo cognitivo na execução de tarefas cotidianas, tampouco restrição da participação social do indivíduo, e os testes de triagem cognitiva são normais. Essas repercussões funcionais do envelhecimento fisiológico se denominam senescência².

Se o declínio cognitivo é mais acentuado do que o esperado para a idade e nível educacional do paciente, ocorrendo comprometimento da memória, com preservação das funções cognitivas globais, e sem limitar a execução das atividades de vida diária, esse quadro caracteriza o comprometimento cognitivo leve (CCL). O CCL está presente em 3\% a 19\% dos adultos acima de 65 anos. Algumas pessoas com essa síndrome podem manter-se estáveis ou retornar ao estado cognitivo normal ao longo do tempo, porém mais da metade evolui para demência dentro de cinco anos. Portanto, o CCL pode ser considerado um fator de risco para demência e seu diagnóstico e tratamento precoces podem beneficiar idosos em estádio anterior à manifestação plena da demência ${ }^{3}$.

Quando múltiplos déficits cognitivos estão presentes, comprovados por testes cognitivos, e suficientes para impedir a realização de atividades de vida diária, é feito o diagnóstico de incapacidade cognitiva. Esta pode ser reversível, como nos casos de delirium e de depressão, ou irreversível, na síndrome demencial e de doenças mentais, como esquizofrenia, paralisia cerebral, parafrenia, entre outras ${ }^{4}$.

De acordo com os critérios da Classificação Internacional de Doença, 10a edição (CID-10) e do Diagnostic and Statistical Manual of Mental Disorders, IV edição (DSM-IV)', a de- mência caracteriza-se por declínio intenso da memória e de outras funções corticais superiores, como linguagem, praxia, capacidade de reconhecer e identificar objetos, abstração, organização, capacidade de planejamento e sequenciamento 7 . No Brasil, segundo dados de 1998, a prevalência de demência em pessoas com mais de 65 anos era de $7,1 \%$, enquanto nas acima de 80 anos era de $20 \% 8$. A cada cinco anos, após os 60 anos, a prevalência da doença dobra, podendo chegar a 50\% nos pacientes com mais de 85 anos $^{4}$.

Doença de Alzheimer, demência vascular, demência com corpos de Lewy e demência frontotemporal são as quatro causas mais frequentes de demência na prática clínica. Cada um desses quadros tem suas características próprias e relaciona-se com fatores biológicos, sociais e patológicos ${ }^{9-13}$.

Para firmar o diagnóstico sindrômico de demência, é necessária avaliação objetiva da função cognitiva, além do desempenho em atividades de vida diária4. O Miniexame do Estado Mental (MEEM) é um teste clínico capaz de rastrear comprometimento e declínio do estado cognitivo ${ }^{14}$. Apesar de, isoladamente, não se prestar ao diagnóstico de demência, o MEEM pode detectar quadros de perda cognitiva, diferenciando do processo de senescência, que predizem o desenvolvimento da síndrome demencial ${ }^{3,15}$.

São vários os impactos sociais e econômicos da demência e, nesse sentido, é de grande importância a determinação de fatores associados ao seu desenvolvimento, possibilitando o reconhecimento de ferramentas úteis na prevenção e atraso na evolução dessa síndrome.

Entre os agentes relacionados com os processos mentais, tem sido evidenciada a prática de atividades físicas e mentais para reduzir o risco do desenvolvimento de demência ${ }^{16,17}$.

As hipóteses sugeridas para explicar a influência de atividades de lazer na redução da perda cognitiva são o estímulo à neurogênese e à sinaptogênese produzida por fatores neurotróficos, que teriam sua produção aumentada durante a prática regular dos exercícios, e a possível ampliação da reserva cognitiva, que ocorreria particularmente em idosos com manutenção de atividades com maior estímulo cognitivo por longo período. Entretanto, não há consenso sobre qual das modalidades de atividades, físicas ou mentais, exercem efeitos mais pronunciados no atraso ou na prevenção da perda cognitiva na população idosa, ou se esses efeitos são semelhantes ${ }^{16,17}$.

Este trabalho visa a verificar se existe influência da prática de estímulo físico ou mental em idosos no risco de demência e se esses estímulos contribuem de maneira semelhante para a prevenção ou redução de déficits cognitivos.

\section{MÉTODOS}

Este estudo foi aprovado pelo Comitê de Ética em Pesquisa da UFMG (Protocolo de Aprovação no 481/08) e pesquisou 
393 pessoas com mais de 80 anos de idade, residentes na região metropolitana de Belo Horizonte (MG).

Os pesquisadores realizaram as entrevistas, no período de fevereiro de 2008 a março de 2009, em domicílios, centros esportivos, asilos, instituições educacionais e grupos de terceira idade.

Após concordância com o termo de consentimento livre e esclarecido, os idosos foram avaliados em seu estado de saúde, uso de medicamentos, bem como em seu estado emocional, estado cognitivo e condições nutricionais. Verificou-se o grau de atividade física e mental. Para tais fins, utilizaram-se questionários validados em outros estudos ${ }^{18-21}$.

Para alcançar os objetivos do estudo, 303 idosos, que atendiam aos critérios do trabalho, foram distribuídos em três grupos:

- grupo 1 (n 101): 50 homens e 51 mulheres que não praticavam atividade mental ou física produtivas;

- grupo 2 (n 100): 50 homens e 50 mulheres praticantes de atividade física rotineira, mas sem atividade mental produtiva;

- grupo 3 (n 102): 52 homens e 50 mulheres que preservavam atividade mental produtiva, mas não praticavam atividade física rotineira.

Para identificar o grupo praticante de atividades físicas rotineiras, foi utilizado o questionário IPAQ (Questionário Internacional de Atividade Física), validado para idosos e modificado para a realidade brasileira ${ }^{18,19}$. Dessa forma, os entrevistados enquadrados no grupo 2 deveriam praticar exercícios, desde os 65 anos, pelo menos três vezes por semana, durante no mínimo uma hora por vez, incluindo: caminhadas, musculação, ginástica localizada, esportes, ioga, corrida, fisioterapia, hidroginástica etc.

A atividade mental produtiva foi avaliada por um questionário elaborado e classificado de acordo com estudos de Sturman et al. ${ }^{20} \mathrm{e}$ Wilson et al. ${ }^{21}$, igualmente modificado. Com isso, foi definido que os entrevistados enquadrados no grupo 3 deveriam exercer sua função desde os 65 anos, pelo menos três vezes por semana, mais de três horas por dia, incluindo: ler jornais e livros; escrever; participar de jogos que requeiram atividade intelectual; participar de grupos de discussão; fazer palavras cruzadas; frequentar, com participação ativa, eventos culturais e cursos etc.

Durante as entrevistas, foi pesquisada a presença de doenças, que poderiam interferir no resultado do trabalho, e investigado o uso de drogas com efeitos psicotrópicos ${ }^{13}$. Para minimizar viés de memória e de falsa resposta, o cônjuge ou um membro da família acompanhou a maioria dos entrevistados ou foi contatado para confirmação das informações.

Foram excluídos 82 voluntários que apresentaram fatores intervenientes a este trabalho. Os critérios para a exclusão basearam-se em: demência diagnosticada, idosos usuários crônicos de medicamentos com ação psicotrópica, distúrbios endocrinometabólicos não compensados, etilismo crônico, desnutrição, encefalopatias e neuropatias, além de limitações psíquicas graves, como alterações de nível de consciência e comprometimento global das funções cognitivas $^{8-10,12,13}$. Tais limitações são predominantemente causadas, no idoso, pela distimia, depressão maior e delirium 8 . Foram eliminadas também as pessoas que estavam em tratamento de afecções que pudessem interferir na atividade física normal, como insuficiência cardíaca, insuficiência respiratória, distúrbios digestivos graves, entre outras.

Para avaliação nutricional, foi utilizada a Triagem Nutricional Subjetiva, validada por Lipschitz em 199422. Esse questionário é composto de perguntas simples, sobre medidas antropométricas, informações dietéticas e avaliação global (saúde global, medicação, estado funcional e autopercepção da nutrição) totalizando 21 pontos. Os idosos que obtiveram 3 a 5 pontos foram considerados de risco nutricional moderado, e os idosos que obtiveram 6 ou mais pontos foram considerados de alto risco nutricional. Os idosos que apresentaram risco nutricional moderado ou alto foram excluídos do trabalho.

Para avaliação de transtornos de humor, foi utilizada a versão brasileira da GDS-15 (Escala de Depressão em Geriatria-15). O diagnóstico de depressão deve ser suspeitado em idosos com seis ou mais respostas positivas para depressão entre os 15 itens da GDS-1511. Quando houve suspeita de depressão, o idoso foi excluído da pesquisa.

O estado cognitivo dos voluntários foi verificado por meio do Miniexame Mental de Folstein ${ }^{14}$, modificado para o Brasil, que se mostrou um instrumento eficaz, em estudos populacionais, para detecção de quadros de perda cognitiva. Esse teste tem sido utilizado também em ambientes clínicos, para o seguimento de quadros demenciais e no controle de resposta ao tratamento ${ }^{15,23}$.

O MEEM é um teste de rastreamento clínico composto por um conjunto de questões que avaliam a orientação para o tempo e o lugar; a memória imediata e de evocação; a atenção e concentração; a linguagem, nomeação e compreensão e a habilidade construcional. O resultado máximo total é de 30 pontos e são necessários cerca de 10 minutos para a sua aplicação.

O risco aumentado de demência foi definido como a presença de perda cognitiva, estabelecida com a obtenção de uma pontuação, no MEEM, inferior à esperada para um indivíduo saudável.

Em nosso meio, foi observada clara e forte influência da escolaridade sobre os escores totais do MEEM. Portanto, foram utilizados diferentes pontos de corte, de acordo com a escolaridade, na definição do risco de demência.

Essa pontuação de corte, em relação à pontuação total de 30 pontos, foi: 29 pontos para pessoas com 11 anos ou mais de estudo; 28 pontos para aqueles que estudaram por um período de 9 a 11 anos; 26,5 para escolaridade de 5 a 8 anos; 25 pontos para os indivíduos que cursaram até 4 anos a escola e 20 pontos para os analfabetos ${ }^{23}$. 
Dessa maneira, reduziu-se a influência do nível de escolaridade como fator de risco para demência. Os idosos que não alcançaram essa pontuação foram considerados portadores de risco aumentado de demência.

Os dados foram apresentados na forma de média e desvio-padrão da média. Para verificar a comparabilidade dos dados em relação à idade, utilizou-se o teste de normalidade de Kolmogorov-Smirnov (KS) e, a seguir, a análise de variância (ANOVA), seguida do pós-teste de Tukey caso os valores fossem diferentes.

A análise da influência do sexo no desempenho no MEEM e do risco de demência entre os diferentes grupos foi realizada por meio do software Epi Info (versão 3.4.3), utilizando-se os testes de associação do qui-quadrado ( $x 2$ ). Em cada grupo foi definido o número de pessoas que apresentaram risco aumentado de demência, ou seja, não obtiveram a pontuação mínima normal no MEEM, de acordo com a escolaridade, e calculou-se o risco relativo com os respectivos intervalos de confiança. Os valores foram considerados significativos para $\mathrm{p}<0,05$.

\section{RESULTADOS}

A amostra foi composta de 152 pessoas do sexo masculino e 151 do sexo feminino, com idade mínima de 80 anos e máxima de 99 anos. A média de idade entre os idosos praticantes de atividade mental foi de $84,9 \pm 4,2$ para o sexo masculino e de $84,0 \pm 3,8$ para o sexo feminino. Para os idosos praticantes de atividade física, a média de idade foi de 84,3 \pm 3,7 para o sexo masculino e de $84,7 \pm 4,4$ para o sexo feminino. Entre os idosos que não praticavam atividade física ou mental produtivas a média de idade no sexo feminino foi de $84,9 \pm 3,9$ e de $84,9 \pm 3,94$ no sexo masculino.
Analisando as características sociodemográficas, foi observado que, do total de participantes, $174(57,4 \%)$ tinham de 80 a 84 anos, 91 (30\%), de 85 a 89 anos e 38 (12,5\%), 90 anos ou mais. Dentre eles, $123(40,6 \%)$ eram casados, 48 (15,8\%), solteiros e 132 (43,6\%), viúvos. Nessa amostra, 29 (9,6\%) indivíduos eram ativos profissionalmente e $274(90,4 \%)$ eram aposentados. Quanto à escolaridade, 9 (3\%) dos idosos eram analfabetos, 104 (34,3\%) cursaram entre 1 e 4 anos de estudos, 119 (39,3\%), entre 5 e 11 anos e $70(23,1 \%)$ possuíam formação superior.

Dentre os idosos praticantes de atividade mental, $57(55,8 \%)$ tinham de 80 a 84 anos, 34 (33,3\%), de 85 a 89 anos e 11 (10,7\%), 90 anos ou mais. Dentre eles, 53 (52\%) eram casados, 12 (11,7\%) eram solteiros e 37 (36,2\%) eram viúvos. Nesse grupo, 13 (12,7\%) eram ativos na profissão e 89 (87,3\%) eram aposentados. Quanto à escolaridade, $12(11,8 \%)$ estudaram de 1 a 4 anos, 50 (49\%), entre 5 e 11 anos e 40 (39,2\%) cursaram o ensino superior.

Dos idosos praticantes de atividade física, 62 (62\%) tinham de 80 a 84 anos, 25 (25\%), de 85 a 89 anos e 13 (13\%), 90 anos ou mais. Dentre eles, 43 (43\%) eram casados, 13 (13\%) eram solteiros e 44 (44\%) eram viúvos. Dentre esses participantes, 10 (10\%) eram ativos na profissão e 90 (90\%) eram aposentados. Quanto à escolaridade, 2 (2\%) eram analfabetos, 36 (36\%) possuíam de 1 a 4 anos de estudos, 38 (38\%), de 5 a 11 anos e 24 (24\%) tinham formação superior.

Por fim, dentre os idosos que não praticavam atividade física ou mental produtivas, 55 (54,4\%) tinham de 80 a 84 anos, 32 (31,6\%), de 85 a 89 anos e 14 (13,8\%), 90 anos ou mais. Dentre eles, $27(26,7 \%)$ eram casados, 23 (22,8\%) eram solteiros e 51 (50,5\%) eram viúvos. Dentre eles, $6(5,9 \%)$ eram ativos na profissão e 95 $(94,1 \%)$ eram aposentados. Quanto à escolaridade, 7 (6,9\%) eram analfabetos, $56(55,4 \%)$ cursaram de 1 a 4 anos de estudo, 31 (30,7\%), de 5 a 11 anos e 6 (6\%) tinham ensino superior (Tabela 1).

Tabela 1. Dados sociodemográficos

\begin{tabular}{|c|c|c|c|c|c|c|c|c|}
\hline \multirow[t]{2}{*}{ Variáveis } & \multicolumn{2}{|c|}{ Amostra total } & \multicolumn{2}{|c|}{ Atividade física } & \multicolumn{2}{|c|}{ Atividade mental } & \multicolumn{2}{|c|}{ Nenhuma atividade } \\
\hline & N Total & $\%$ & N Total & $\%$ & N Total & $\%$ & N Total & $\%$ \\
\hline Participantes & 303 & 100 & 100 & 33 & 102 & 33,6 & 101 & 33,3 \\
\hline \multicolumn{9}{|l|}{ Sexo } \\
\hline Masculino & 152 & 50,1 & 50 & 50 & 52 & 50,9 & 50 & 49,5 \\
\hline Feminino & 151 & 49,8 & 50 & 50 & 50 & 49 & 51 & 50,4 \\
\hline \multicolumn{9}{|l|}{ Idade (em anos) } \\
\hline 80 a 84 & 174 & 57,4 & 62 & 62 & 57 & 55,8 & 55 & 54,4 \\
\hline 85 a 89 & 91 & 30 & 25 & 25 & 34 & 33,3 & 32 & 31,6 \\
\hline 90 ou mais & 38 & 12,5 & 13 & 13 & 11 & 10,7 & 14 & 13,8 \\
\hline \multicolumn{9}{|l|}{ Estado civil } \\
\hline Casado & 123 & 40,6 & 43 & 43 & 53 & 52 & 27 & 26,7 \\
\hline Solteiro & 48 & 15,8 & 13 & 13 & 12 & 11,7 & 23 & 22,8 \\
\hline Viúvo & 132 & 43,6 & 44 & 44 & 37 & 36,2 & 51 & 50,5 \\
\hline \multicolumn{9}{|l|}{ Profissão } \\
\hline Ativo & 29 & 9,6 & 10 & 10 & 13 & 12,7 & 6 & 5,9 \\
\hline Aposentado & 274 & 90,4 & 90 & 90 & 89 & 87,3 & 95 & 94,1 \\
\hline \multicolumn{9}{|l|}{ Escolaridade } \\
\hline Analfabeto & 9 & 3 & 2 & 2 & 0 & 0 & 7 & 6,9 \\
\hline 1 a 4 anos & 104 & 34,3 & 36 & 36 & 12 & 11,8 & 56 & 55,4 \\
\hline 5 a 11 anos & 119 & 39,3 & 38 & 38 & 50 & 49 & 31 & 30,7 \\
\hline Superior & 70 & 23,1 & 24 & 24 & 40 & 39,2 & 6 & 6 \\
\hline
\end{tabular}


Na análise da idade, foi atendida a suposição de normalidade em todos os grupos. Comparando-se a idade entre os grupos, não houve diferença na análise de variância $(p=0,292)$.

Comparando o número de indivíduos com risco aumentado de demência, em relação ao sexo, dentro de cada grupo, o grupo 1 apresentou $p=0,558$; o grupo $2, p=0,531$ e o grupo $3, p=0,180$, indicando que o sexo não se relacionou com o desempenho no teste.

Houve diferença entre os grupos $(p<0,001)$ na comparação da porcentagem de idosos com risco aumentado de demência. Este foi maior no grupo 1, com porcentagem de $84,2 \%$ de risco aumentado de desenvolver demência. Por outro lado, o grupo 2 teve $65 \%$ de risco aumentado de demência. Já no grupo 3 esse risco foi de 32,4\% (Tabela 2).

Tabela 2. Risco aumentado de demência em idosos*

\begin{tabular}{lcccc}
\hline \multirow{2}{*}{ Grupos } & Idosos pesquisados & & \multicolumn{2}{c}{ Idosos com risco de demência } \\
\cline { 2 - 4 } \cline { 5 - 5 } & N & & N & $\%$ \\
\hline Grup0 1 & 101 & & 85 & 84,2 \\
Grup0 2 & 100 & & 65 & 65 \\
Grupo 3 & 102 & & 33 & 32,4 \\
Total & 303 & & 183 & 60,4 \\
\hline
\end{tabular}

* Risco aumentado de demência: idosos com pontuação abaixo do limite de normalidade no Miniexame do Estado Mental, considerando a escolaridade.

Comparando as incidências de risco aumentado de demência entre os grupos, os indivíduos do grupo 1 apresentaram risco relativo de 2,21 (95\% IC: 1,31-3,73), quando comparados aos indivíduos do grupo 2, e 4,27 (95\% IC: 2,67-6,83), quando comparados aos do grupo 3 . O grupo 2 apresentou risco relativo de 1,93 (95\% IC: 1,43-2,61) em relação ao grupo 3 (Tabela 3).

Tabela 3. Risco relativo para o desenvolvimento de demência entre os grupos

\begin{tabular}{lll}
\hline Grupos & Risco relativo & IC95\%* \\
\hline Grupo 1 x Grupo 2 & 2,21 & $1,31-3,73$ \\
Grupo 1 x Grupo 3 & 4,27 & $2,67-6,83$ \\
Grupo 2 × Grup0 3 & 1,93 & $1,43-2,61$ \\
\hline
\end{tabular}

*IC: intervalo de confiança.

\section{DISCUSSÃO}

Este estudo mostrou associação entre a prática de atividades físicas e mentais e diminuição da perda cognitiva em idosos, reduzindo, consequentemente, o risco do desenvolvimento de demência. Entretanto, a prática isolada de atividade mental foi fator mais eficaz em minimizar o déficit cognitivo do que a de atividade física.

Ainda não há um consenso, na literatura, sobre os resultados presentes neste estudo. Estudos epidemiológicos já demonstraram que há uma associação entre altos índices de atividades de lazer, principalmente envolvendo atividades mentais (como leitura e jogos de tabuleiro), e redução no declínio cognitivo em idosos, exercendo influência no risco de demência ${ }^{24,17}$. Alguns autores evidenciaram que altos níveis de atividades físicas, mentais e sociais associam-se igualmente a um risco de demência reduzido e que a prática simultânea de dois ou dos três componentes exercem efeitos ainda mais pronunciados na redução do risco ${ }^{16}$.

Entretanto, ainda não foi estabelecido se a prática isolada de atividades físicas reduz o risco de demência. Isso pode ser explicado pela dificuldade na separação das atividades físicas sem qualquer estímulo cognitivo, das que possuem um componente cognitivo em sua realização. Quando foi analisada a influência de atividades predominantemente físicas (como caminhada, corrida, ciclismo e natação), não foi demonstrada redução significativa no declínio cognitivo ${ }^{20}$. Porém, a diferença na intensidade e duração do estímulo físico nos diversos estudos pode explicar os diferentes resultados encontrados.

Existem diferentes hipóteses para explicar o mecanismo pelo qual a prática dessas atividades reduz o risco de demência. Dados da literatura ${ }^{25}$ sugerem que o exercício físico produz benefícios para a saúde em geral e também para a função cognitiva, particularmente em indivíduos com idade avançada. Há indícios de aumento nos níveis de fatores neurotróficos cerebrais (FNC) que estimulam a sinaptogênese e a neurogênese e aumentam a resistência a agressões cerebrais, melhorando o aprendizado e o desempenho mental, com manutenção da memória de evocação.

Foi evidenciado, em camundongos, que a atividade física é capaz de afetar os níveis de hormônio esteroide e estresse, responsáveis por influenciar na aprendizagem, neurogênese e fatores de plasticidade neural como formação, estabilidade, especificidade sináptica e reversibilidade ${ }^{26}$.

A prática de atividade mental produz efeitos mais pronunciados, provavelmente por induzir maior estímulo cerebral, promovendo a neurogênese. De acordo com trabalhos de Churchill et al.27, o estímulo mental específico aumenta seletivamente a sinaptogênese em adultos, enquanto o exercício físico aumenta, além da neurogênese, componentes não neuronais do cérebro, como sua vascularização, exercendo um efeito menos específico ${ }^{25}$.

Outro benefício das atividades mental e física na redução do risco de demência é o estímulo social durante sua prática ${ }^{25}$.

Além disso, o possível efeito benéfico da estimulação cognitiva supõe a existência de uma reserva cognitiva que pode ser potencialmente utilizada. Para demência, essa hipótese de reserva foi proposta primeiramente por Katzman ${ }^{28}$ e desenvolvida por Stern ${ }^{29}$. Ela sugere que há uma capacidade cerebral de recuperação perante os danos neuropatológicos relacionados ao envelhecimento. Essa capacidade pode ser potencializada por uso mais eficiente das redes cerebrais e melhor habilidade de recrutar redes alternativas quando necessário. Portanto, o aumento da reserva cognitiva, por meio da prática de atividades de estímulo cognitivo, pode levar a um atraso das manifestações clínicas relacionadas à demência. 
Fatores genéticos podem estar envolvidos no aparecimento da demência e na sua velocidade de progressão. $\mathrm{Na}$ doença de Alzheimer, por exemplo, já é bem documentada a influência de alelos específicos do gene da apolipoproteína E e butirilcolinesterase-K, além de mutações no cromossoma 12 no aparecimento desse tipo de demência ${ }^{30}$. Portanto, é possível que haja predisposição genética envolvida também na gênese de outros tipos de demência. Nessas situações, fatores ambientais, como a prática de atividade física ou mental, podem alterar apenas em parte a progressão da doença.

Sintomas de depressão e de ansiedade também estão associados à deterioração da memória e de outras funções $\operatorname{cognitivas}^{4,10}$. Na tentativa de reduzir o risco dessa interferência, este estudo buscou eliminar os participantes com suspeita de depressão ou distimia, mediante a versão brasileira da GDS-1511,19. No entanto, esse é um teste de triagem, não particulariza cada indivíduo, estando, portanto, sujeito a faIhas, além de não avaliar o estado de ansiedade.

Esse trabalho possui algumas limitações. É possível que os idosos pesquisados praticassem atividade física por serem previamente saudáveis ou como tratamento de uma afecção física prévia. Por outro lado, os idosos não praticantes de exercício físico poderiam estar limitados por deficiências físicas ou mentais prévias que impedissem a prática de atividades físicas. Para esse estudo, os pesquisadores utilizaram como critérios de exclusão de indivíduos da pesquisa dados colhidos durante a entrevista, como histórico médico pregresso, uso de medicamentos e resposta aos questionários de rastreamento nutricional e de transtornos de humor. Portanto, não foi realizado diretamente o diagnóstico das afecções que porventura existissem.

\section{CONCLUSÃO}

Os resultados do presente estudo indicam que a prática regular de atividades mentais e físicas retarda o declínio cognitivo, reduzindo o risco de demência. Dentre essas atividades, as mentais foram mais eficazes.

\section{REFERÊNCIAS}

1. IBGE. Instituto Brasileiro de Geografia e Estatística. Síntese de indicadores sociais 2006. Rio de Janeiro: 2006. 317p. (Estudos \& Pesquisas - Informação demográfica e socioeconômi(a, 19). Disponível em: <http://www.ibge.gov.br>. Acessado em: 2 Jul 2010.

2. Small SA. Age-related memory decline - Current concepts and future directions. Arch Neurol. 2001;58(3):360-4.

3. Gauthier S, Reisberg B, Zaudig M, Petersen RC, Ritchie K, Broich K, et al. Mild cognitive impairment. Lancet. 2006;367(9518):1262-70.

4. Moraes EM, organizador. Princípios básicos de geriatria e gerontologia. 1a ed. Belo Horizonte: Coopmed; 2008. p. 700.
5. World Health Organization. The ICD-10 Classification of Mental and Behavioural Disorders, Geneva; 1992.

6. American Psychiatry Association. Diagnostic Criteria from DSM-IV. Washington; 1994

7. Herrera E, Caramelli P, Silveira ASB, Nitrini R. Epidemiologic survey of dementia in a community-dwelling Brazilian population. Alzheimer Dis Assoc Disord. 2002;16(2):103-8.

8. Lopes MA, Bottino CMC. Prevalência de demência em diversas regiões do mundo: análise dos estudos epidemiológicos de 1994 a 2000. Arq Neuropsiquiatr. 2002;60(1):61-9.

9. Lee KS, Cheong HK, Kim EA, Kim KR, Oh BH, Hong CH. Nutritional risk and cognitive impairment in the elderly. Arch Gerontol Geriatr. 2009;48(1):95-9.

10. Potter GG, Steffens DC. Contribution of depression to cognitive impairment and dementia in older adults. Neurologist. 2007;13(3):105-17.

11. Almeida OP, Almeida SA. Confiabilidade da versão brasileira da escala de depressão em geriatria (GDS) versão reduzida. Arq Neuropsiquiatr. 1999;57(2B):421-6.

12. Osimani A, Berger A, Friedman J, Porat-Katz BS, Abarbanel JM. Neuropsychology of vitamin B12 deficiency in elderly dementia patients and control subjects. J Geriatr Psychiatry Neurol. 2005;18(1):33-8.

13. Gray SL, Lai KV, Larson EB. Drug-induced cognition disorders in the elderly - Incidence, prevention and management. Drug Safety. 1999;21(2):101-22.

14. Folstein MF, Folstein SE, McHugh PR. Mini-Mental State: a practical method for grading the cognitive state of patients for clinician. J Psychiatr Res. 1975;12:189-98.

15. Ercoli LM, Siddarth P, Dunkin JJ, Bramen J, Small GW. MMSE items predict cognitive decline in persons with genetic risk for Alzheimer's disease. J Geriatr Psychiatry Neurol. 2003;16(2):67-73.

16. Karp A, Paillard-Borg S, Wang HX, Silverstein M, Winblad B, Fratiglioni L. Mental, physical and social components in leisure activities equally contribute to decrease dementia risk. Dement Geriatr Cogn Dis. 2006;21(2):65-73.

17. Verghese J, Lipton RB, Katz MJ, Hall CB, Derby CA, Kuslansky G, et al. Leisure activities and the risk of dementia in the elderly. N Engl J Med. 2003;348(25):2508-16.

18. Benedetti TRB, Antunes PC, Rodriguez-Añez CR, Mazo GZ, Petroski EL. Reprodutibilidade e validade do Questionário Internacional de Atividade Física (IPAQ) em homens idosos. Rev Bras Med Esporte. 2007;13:11-6.

19. Benedetti TRB, Mazo GZ, Barros MVG. Aplicação do Questionário Internacional de Atividade Física para avaliação do nível de atividade física de mulheres idosas: validade concorrente e reprodutibilidade teste-reteste. R Bras Ciên e Mov. 2004;12(1):25-34.

20. Sturman MT, Morris MC, De Leon CFM, Bienias JL, Wilson RS, Evans DA. Physical activity, cognitive activity, and cognitive decline in a biracial community population. Arch Neurol. 2005;62(11):1750-4.

21. Wilson RS, Leon CFM, Barnes LL, Schneider JA, Bienias JL, Evans DA, et al. Participation in cognitively stimulating activities and risk of incident Alzheimer disease. JAMA. 2002; 287(6):742-8.

22. Lipschitz DA. Screening for nutritional status in the elderly. Prim Care. 1994;21(1):55-67.

23. Brucki SMD, Nitrini R, Caramelli P, Bertolucci PHF, Okamoto IH. Sugestões para 0 uso do Miniexame do Estado Mental no Brasil. Arq Neuropsiquiatr. 2003;61(3B):777-81.

24. Akbaraly TN, Portet F, Fustinoni S, Dartigues JF, Artero S, Rouaud 0, et al. Leisure activities and the risk of dementia in the elderly results from the Three-City Study. Neurology. 2009;73(11):854-61.

25. Fratiglioni L, Paillard-Borg S, Winblad B. An active and socially integrated lifestyle in late life might protect against dementia. Lancet Neurol. 2004;3(6):343-53.

26. Van Praag H, Christie BR, Sejnowski TJ, Gage FH. Running enhances neurogenesis, learning, and long-term potentiation in mice. Proc Natl Acad Sci U S A. 1999;96(23):13427-31.

27. Churchill JD, Galvez R, Colcombe S, Swain RA, Kramer AF, Greenough WT. Exercise, experience and the aging brain. Neurobiol Aging. 2002;23(5):941-55.

28. Katzman R. Education and the prevalence of dementia and Alzheimer's disease. Neurology. 1993;43:13-20.

29. Stern Y. What is cognitive reserve? Theory and research application of the reserve concept. J Int Neuropsychol. 2002;8:448-60.

30. Alves TCTF, Wajngarten M, Busato GF. Fatores de risco cardiovascular, declínio cognitivo e alterações cerebrais detectadas através de técnicas de neuroimagem. Rev Psiq Clín. 2005;32(3):160-9. 